\title{
Monitoring of upper oesophageal sphincter pressure in children
}

\author{
G P Davidson, J Dent, J Willing
}

\begin{abstract}
In children technical limitations of upper oesophageal sphincter manometry have restricted investigation to the pull through technique under sedation. In this study we have used an adapted sleeve manometric technique for upper oesophageal sphincter monitoring in unsedated children and determined the influence of the state of arousal on upper oesophageal sphincter pressure. Twenty six children aged 3 to 42 months (median 17.5 months), who were referred for evaluation of oesophageal motor function, were studied with dual sleeve manometric assemblies which monitored upper and lower oesophageal sphincter pressures simultaneously. Pharyngeal, oesophageal body, and gastric pressures were also monitored with seven perfused side holes. Recordings were made for four hours after a meal and were technically successful in $\mathbf{2 4}$ children. The child's state of arousal was scored every 12th minute as follows: (A) resting, eyes closed, (B) resting, eyes open, (C) moving but comfortable, (D) restless and uncomfortable, (E) crying. In $67 \%$ of the 12 minute samples the children showed good adaptation to the procedure (arousal states A to C). There was a highly significant difference in upper oesophageal sphincter pressure between each of the arousal states $(\mathbf{p}<0.0001)$, being lowest in category $A$ at (mean (SD) 18.1 (10.3) $\mathrm{mmHg}$ and highest in category D 55.7 (13.2) mmHg. Abrupt changes in the state of arousal were associated with equally abrupt changes in upper oesophageal sphincter pressure. The state of arousal of unsedated children has an important influence on upper oesophageal sphincter pressure. It is essential that this factor is controlled for in any studies of upper oesophageal sphincter tone in children. The sleeve technique is capable of monitoring upper oesophageal sphincter motility for prolonged periods in unsedated children.
\end{abstract}

Gastroenterology Unit, Adelaide Children's Hospital, North

Adelaide, South Australia 5006

G P Davidson

J Willing

Gastroenterology Unit,

Royal Adelaide Hospital,

Adelaide, South Australia

5000

J Dent

Correspondence to:

Dr G P Davidson

Grastroenterology Unit,

Adelaide Children's Hospital

King William Road, North

Adelaide, South Australia
5006 .

Accepted for publication

20 August 1990

dicates that upper oesophageal sphincter mobility results in frequent displacement of stationed side hole from the zone of maximal pressure. ${ }^{45}$ Consequently, the pull through technique has been used extensively to overcome the problem of displacement, but this approach gives only a brief sampling of upper oesophageal sphincter pressure. These limitations mean that the side hole technique is incapable of giving accurate longterm measurements of sphincter pressure.

The evaluation of upper oesophageal sphincter function in children poses additional technical demands when compared with upper oesophageal sphincter manometry in adults. Children are rarely capable of coping calmly with the discomforts caused by the frequent manometric catheter manipulations required for both pull through and stationed side hole manometric recordings. These difficulties are underscored by the fact that there are only two reports of studies of sphincter function in children in which perfused manometric methods have been used. ${ }^{67}$ Sedation was used in both of these studies from which only limited conclusions could be drawn about upper oesophageal sphincter function. The use of sedation may have had a substantial influence on values obtained, since studies of sphincter function in adults and animals indicate that sleep ${ }^{8}$ and anaesthesia ${ }^{9}$ appreciably lower upper oesophageal sphincter pressure while mental stress leads to increased sphincter contraction. ${ }^{10}$ The possibility that sedation may have influenced values of upper oesophageal sphincter pressure obtained in children has been acknowledged by Sondheimer. ${ }^{6}$

An effective method for the evaluation of upper oesophageal sphincter function is needed in children as they have a high incidence of problems that are thought to arise from defective control of transit across the upper oesophageal sphincter. Oesophagopharyngeal reflux can lead to failure to thrive and recurrent respiratory disease. Furthermore, there has been inadequate definition of the part played by upper oesophageal sphincter dysfunction in swallowing disorders. Recently, sleeve manometry has been adapted and applied successfully to monitoring upper oesophageal sphincter pressure in adults. ${ }^{+}$ This technique allows pressure recording for long periods of time without the need for frequent adjustment of the manometric assembly position. In studies in adults the sleeve has allowed the evaluation of reflex control of the upper oesophageal sphincter, including responses to swallowing ${ }^{5}$ and to oesophageal distension with gas or liquid refluxate." The aim of the present study was to evaluate the use of a miniaturised upper oesophageal sphincter sleeve in unsedated children and to determine the influence of their state of arousal on basal sphincter pressure values.

\section{Patients and methods}

Twenty six consecutively referred children aged 3 to 42 months (median 17.5 months) were enrolled in the study after referral to the Gastro- 
enterology Unit of Adelaide Children's Hospital for evaluation of symptoms thought to be caused by gastro-oesophageal reflux or a swallowing disorder. The major priority in these children was the evaluation of lower oesophageal sphincter and oesophageal body function as an aid to clinical management. These measurements were made with a lower oesophageal sphincter sleeve and side holes. Measurements of upper oesophageal sphincter pressure were made simultaneously with oesophageal body and lower oesophageal sphincter manometry. All children were fully assessed clinically by a consultant paediatric gastroenterologist. The study protocol was approved by the Ethics Research Committee of Adelaide Children's Hospital. Informed parental consent was obtained before the study. The presenting symptoms are given in the Table.

\section{PROTOCOL FOR PH AND MOTILITY MONITORING}

A purpose built manometric assembly which incorporated two sleeve sensors for concurrent monitoring of lower and upper oesophageal sphincter pressures was passed transnasally without the aid of sedation after a three hour fast. Side holes monitored gastric, oesophageal body, and pharyngeal pressures (Fig 1, see below). Distal oesophageal pH was monitored concurrently with a miniature intraluminal glass electrode. After positioning of the $\mathrm{pH}$ electrode and manometric assembly the child was allowed to settle and was then fed appropriately for age with formula or non-acid food such as sandwiches and milk. In the case of children fed formula, this was introduced into the stomach via the manometric assembly if they did not take their usual volume of feed orally. Monitoring of spontaneous patterns of motility and oesophageal $\mathrm{pH}$ was started at the end of the meal and continued for four hours with the children recumbent and unsedated.

\section{RECORDING TECHNIQUE}

Two manometric assemblies were built with different intersleeve distances to cope with the range of intersphincteric distances found in this age group. The nine lumen assemblies had an outer diameter of $3.5 \mathrm{~mm}$. The arrangement of

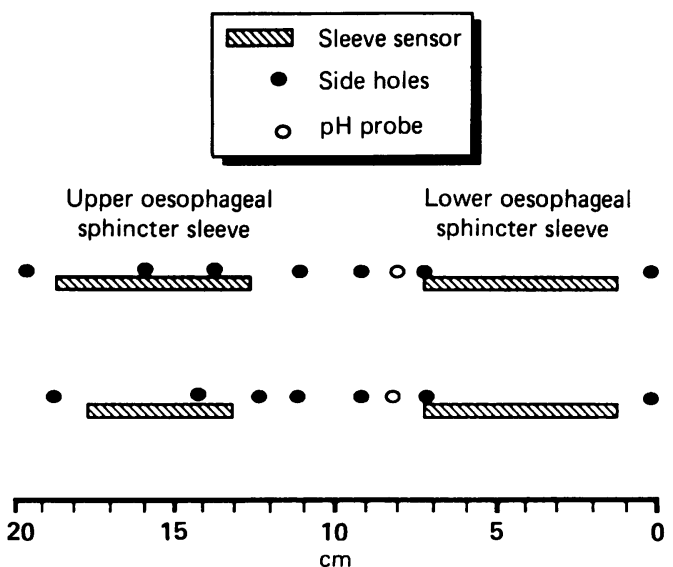

Figure 1: Diagrammatic representation of the two manometric assemblies, showing the position of sleeve sensors, $p H$ electrode, and side holes. the two sleeves and seven side holes in each assembly is shown in Figure 1. The oval cross section of the upper oesophageal sphincter sleeve segment of the assemblies was $5.5 \mathrm{~mm}$ by $3.5 \mathrm{~mm}$. This cross section was chosen to position the upper oesophageal sphincter sleeve in either the anterior or posterior orientation. ${ }^{4} \mathrm{~A}$ low compliance pneumohydraulic pump was used to perfuse sleeves at $0.6 \mathrm{ml} / \mathrm{min}$ and the side holes at $0.3 \mathrm{ml} / \mathrm{min}$ except the pharyngeal side hole channel which was perfused at $0.15 \mathrm{ml} / \mathrm{min}$. The glass $\mathrm{pH}$ probe (Micro-electrodes, New Hampshire, USA, model MI-506) was passed with the manometric assembly, being taped to it $1 \mathrm{~cm}$ above the distal sleeve with a $2 \mathrm{~mm}$ wide band of plastic adhesive. An external skin reference electrode (Micro-electrodes, New Hampshire, USA, model MI-402) was fixed firmly to a limb, and made electrical contact with the skin through a conductive gel which was kept in place with an occlusive dressing.

The data of Strobel $e t a l^{12}$ were used to predict the teeth to lower oesophageal sphincter distance as an aid to correct positioning of the manometric assembly and to choose the catheter with the most appropriate intersleeve distance. The assembly was initially positioned so that the distal sleeve was astride the lower oesophageal sphincter. The assembly position was then adjusted to give effective monitoring of upper oesophageal sphincter pressure with the proximal sleeve, provided that this did not result in loss of correct positioning of the lower oesophageal sphincter sleeve. Even during crying, the characteristic patterns of pressure oscillation seen in the pharynx, oesophagus, and stomach allowed confirmation of correct assembly positioning. The catheter was then firmly anchored to the nose and not readjusted unless absolutely necessary.

Pressure and distal oesophageal $\mathrm{pH}$ were recorded on a 12 channel polygraph (Grass Instrument, Quincy, Mass, USA, model 7D). A chart paper speed of $150 \mathrm{~mm} / \mathrm{min}$ was used to permit subsequent analysis of time relations among pressure events at different recording points. Before each study signals from the pressure transducers (Deseret Medical, Sandy, Utah, USA, model 38-848-1) were set to identical baselines and gains. These settings were checked at the conclusion of measurements and were always stable. The $\mathrm{pH}$ electrode was calibrated before and after each test with $\mathrm{pH} 4$ and 7 buffers.

\section{DATA ANALYSIS}

\section{Assessment of state of arousal}

Every 12th minute during the monitoring period the child's state of arousal was assessed by the same trained observer (JW) who sat with the children throughout each study. The arousal states were marked on the trace as the study proceeded. The classification of arousal state was relatively coarse and designed to allow reliable recognition by simple direct observation of behaviour, as follows: (A) resting with eyes shut, (B) resting with eyes open, (C) moving briefly more than twice a minute but comfortable, (D) restless and uncomfortable, and (E) crying. 
$(\mathrm{mm} \mathrm{Hg})$

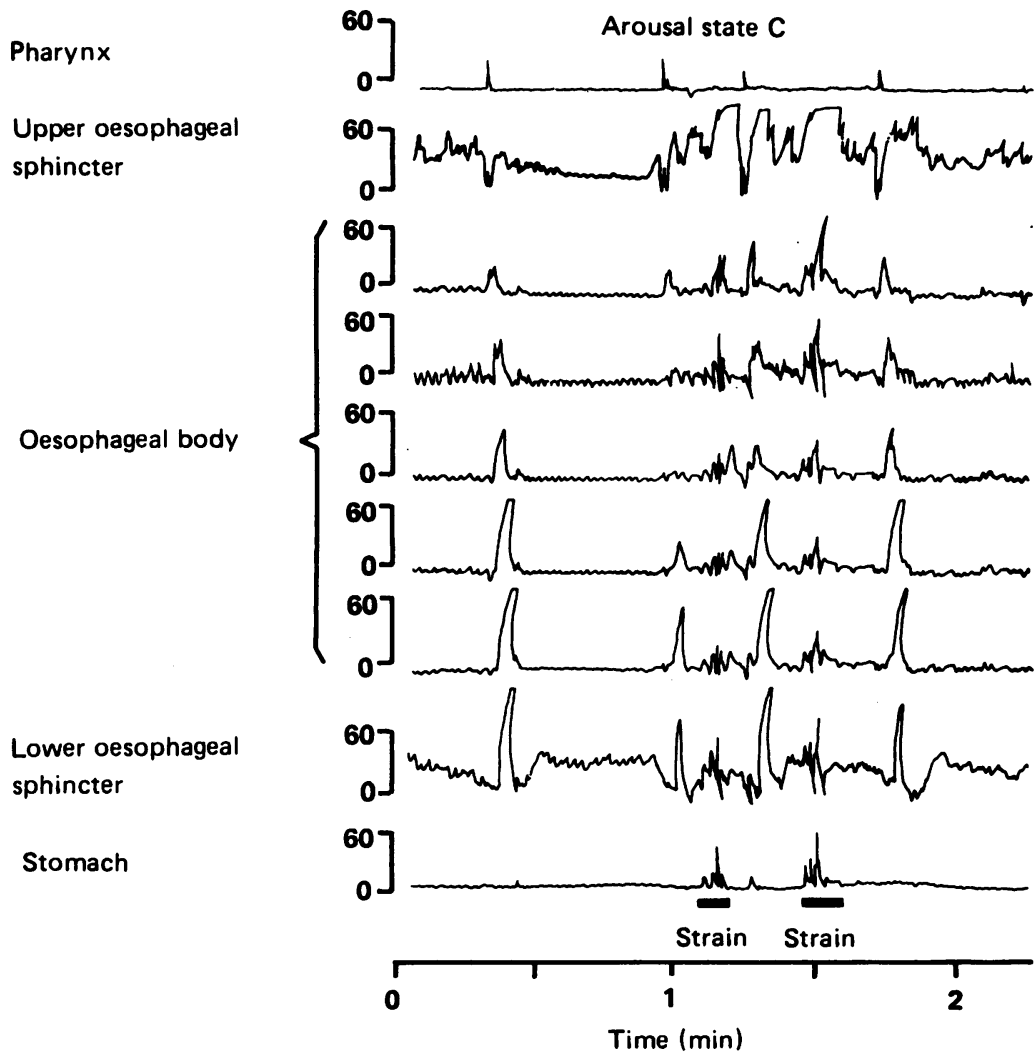

Figure 2: A representative tracing from a 9 month old infant showing the correlation of pharyngeal, oesophageal, and gastric events. Swallowing is indicated by the pressure spikes in the pharyngeal channel. Upper oesophageal sphincter pressure was recorded successfully despite swallowing, straining, and body movement (arousal state $C$ ). The upper oesophageal sphincter tracing shows considerable variability of pressure with pronounced augmentation during straining.
Presenting symptoms in 24 children evaluated

\begin{tabular}{lc}
\hline Symptom & No \\
\hline Irritability/screaming & 15 \\
Vomiting & 15 \\
Abdominal pain & 3 \\
Cough & 2 \\
Food refusal & 3 \\
Pharyngeal incoordination & 1 \\
Apnoea & 1 \\
\hline
\end{tabular}

\section{Results}

POSITIONING THE CATHETER IN THE UPPER OESOPHAGEAL SPHINCTER

Manometric assembly positioning was defined as satisfactory when the side holes at each end of the lower oesophageal sphincter sleeve showed gastric and oesophageal body pressure patterns while the side holes at either end of the upper oesophageal sphincter sleeve indicated pharyngeal and oesophageal body pressures (Fig 2). In two children the intersleeve distance was not compatible with simultaneous, technically satisfactory recording from both the upper and lower oesophageal sphincters. In these children the assembly was positioned to meet the clinical requirement for adequate lower oesophageal sphincter manometry. The results presented below are for the 24 children in whom concurrent monitoring of lower and upper oesophageal sphincter pressures proved possible. In Figure 2 a segment of recording shows the ability of the assembly to record a complete picture of oesophagopharyngeal function.

\section{TOLERANCE AND TECHNICAL SUCCESS OF THE PROCEDURE}

The regular evaluation of the child's state of arousal and the interpretability of the tracings of upper oesophageal sphincter pressure allowed objective assessment of the child's tolerance to the recording procedure; $67 \%$ of the 12 th minute samples were in categories $A$ to $C$, indicating good tolerance of the procedure (Fig 3). In 351 of the total of 48012 th minute samples (73\%) a value could be derived for upper oesophageal sphincter pressure according to the analysis approach described in Methods. Among the 12th minute samples that could not be analysed, crying was the cause in $9 \%$ and excessively frequent swallowing in $13 \%$. In only $2 \%$ of the 12th minute samples was upper oesophageal sphincter pressure uninterpretable because of malposition of the sleeve. Other forms of technical failure accounted for $3 \%$ of uninterpretable values. Missing values of upper oesophageal sphincter pressure for the 12th minute (129/480) could be obtained from the subseqent 13 th or 14 th minutes in 49 instances. This gave a total of $400 / 480$ or $83 \%$ of samples for which there was a value for upper oesophageal sphincter pressure.

A mean value of the data points for each state of arousal was calculated for each patient and the group as a whole. An analysis of variance for repeated measures was performed. The overall mean for a particular arousal category was substituted for any missing values. There was no difference in significance using this method as compared to an analysis of variance run on the eight patients with values in all categories.
EFFECT OF CHANGES OF STATE OF AROUSAL ON UPPER OESOPHAGEAL SPHINCTER PRESSURE

Pronounced changes of upper oesophageal sphincter pressure were seen in association with changes in the state of arousal of the children. 


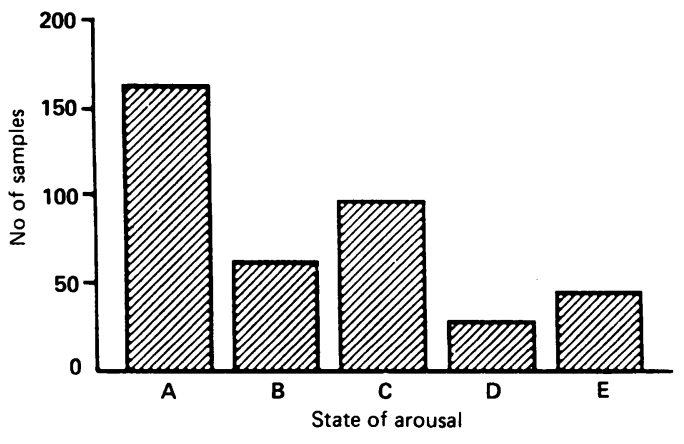

Figure 3: Tolerance of the procedure shown by distribution of categories of state of arousal assessed on every 12 th minute of recording.

Even when the arousal state was stable, basal pressure showed some variation (Figs 2 and 4). Upper oesophageal sphincter pressure was lowest when the child was resting with eyes closed (state A). When there was an abrupt change in the state of arousal there was an associated immediate change of pressure which was often severalfold (Fig 4). Mean (SD) values for upper oesophageal sphincter pressure shown in Figure 5 ranged from $18.0(10.3) \mathrm{mmHg}$ for arousal state $A$ to $55.7(13 \cdot 2) \mathrm{mmHg}$ for state D. Meaningful values could not be derived for state $E$ because of large pressure swings in pharyngeal oesophageal and gastric pressures related to repeated straining and crying. During crying, however, there was a differential and pronounced increase in upper oesophageal sphincter pressure which often resulted in an off scale recording $(>100 \mathrm{mmHg})$. There was a highly significant relation between state of arousal and upper oesophageal sphincter $(\mathrm{p}<0.0001)$.

\section{Discussion}

In this report we describe what we believe to be the first technically successful longterm recordings of upper oesophageal sphincter in unsedated children. This first experience has shown that the level of basal upper oesophageal sphincter pressure is closely linked to the state of arousal, being augmented severalfold if the child is uncomfortable.

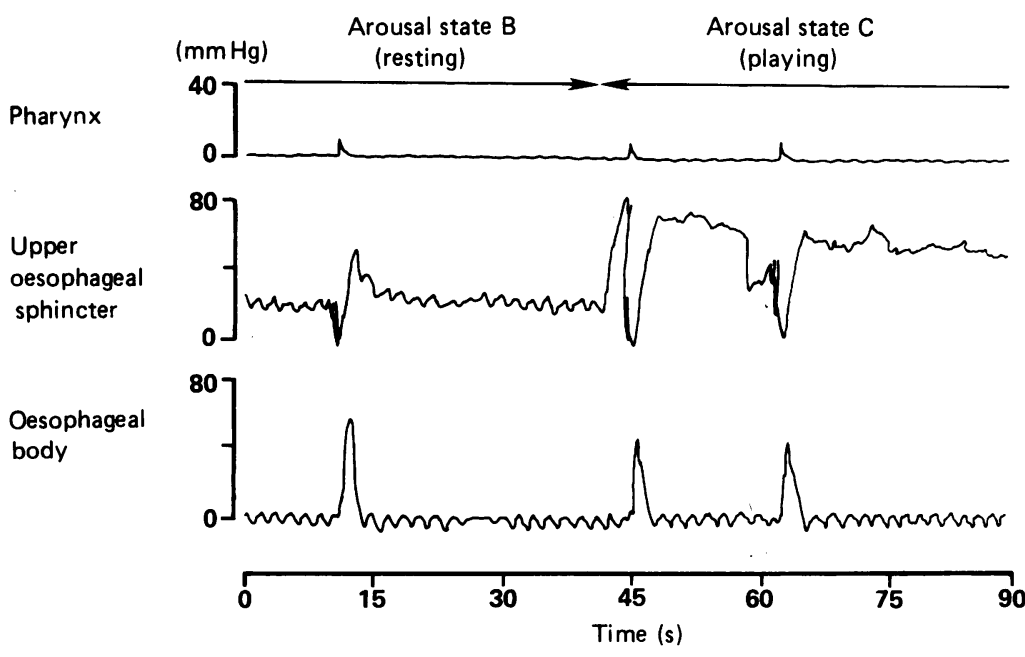

Figure 4: Representative tracing showing an abrupt increase in upper oesophageal sphincter pressure with change from arousal state $B$ to arousal state $C$.

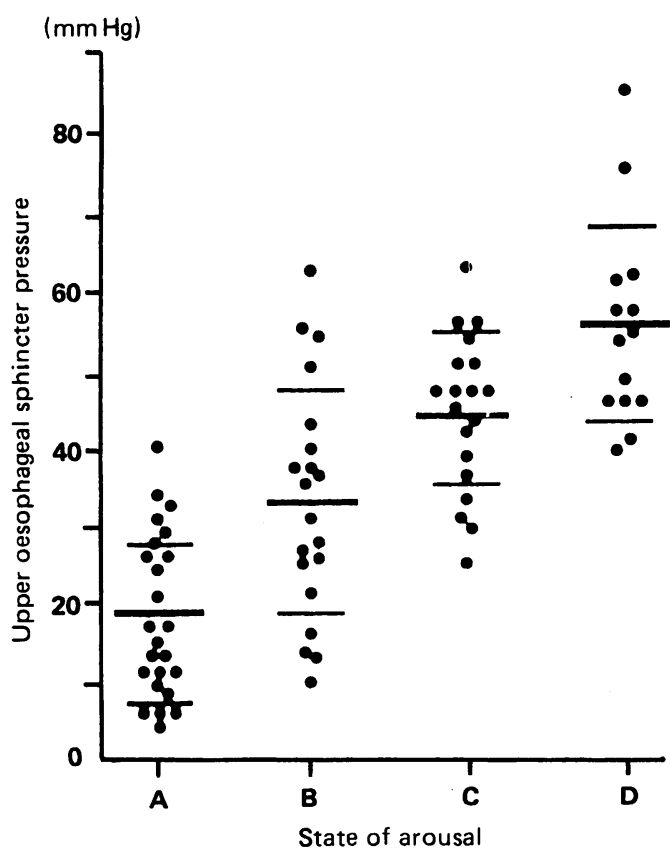

Figure 5: Individual subject and group mean $(S D)$ values of upper oesophageal sphincter pressure for each state of arousal. There is a highly significant $(p<0.0001)$ correlation between state of arousal and pressure.

The finding that the state of arousal has a substantial influence on sphincter pressure has major implications for research on the upper oesophageal sphincter in children. Until now the major emphasis has been on obtaining values of basal upper oesophageal sphincter pressure and to relate these to suspected dysfunction. If regurgitation results from defective basal sphincter tone, previous measurements will have been so noisy that such a defect may not have been recognisable. Our results are consistent with recent observations that mental stress increases upper oesophageal sphincter pressure in adults. ${ }^{10}$ Our finding that upper oesophageal sphincter pressure is highly labile is also consistent with the reports of low basal pressure levels during sleep in adult volunteers ${ }^{8}$ or with anaesthesia in opossums. ${ }^{9}$

Our observation of state of arousal depended on the judgment of a trained observer (JW) who had no preconceived notion of the effects of state of arousal on upper oesophageal sphincter pressure. We found other approaches to evaluation of the state of arousal impractical in the setting of the studies. The measures of arousal were gross and unlikely to be susceptible to major observer error.

Previous studies with the sleeve technique have shown that various forms of sustained abdominal or thoracic straining are associated with similarly sustained increases in upper oesophageal sphincter pressure. ${ }^{13}$ In the present study straining was readily recognisable by rising abdominal and thoracic pressure and was associated with an increase in upper oesophageal sphincter pressure considerably in excess of the increase in thoracic or abdominal pressure (Fig $2)$. This suggests that the increase in upper oesophageal sphincter pressure observed was due at least in part to a real increase in sphincter contraction. Such contractile responses of the sphincter did not, however, explain the pro- 
longed, consistent changes in pressure seen in arousal states $A$ to $D$, as these arousal states were not associated with sustained straining. By comparison, repeated vigorous straining was seen in arousal state $E$ when the children were crying. In these circumstances upper oesophageal sphincter pressure was often so high that it was off scale and so not analysable.

Many factors including straining, valsalva manoeuvre, mental stress, oesophageal distension, and speech can increase the tone of the upper oesophageal sphincter musculature. ${ }^{41013}$ The resting tone of many striated muscles is also increased during states of arousal and it seems that the upper oesophageal sphincter is no exception, especially in children.

We believe that the most promising approach to better definition of the patterns of upper oesophageal sphincter function associated with oesophagopharyngeal reflux is to monitor upper oesophageal sphincter pressure and capture motor events associated with spontaneous occurrence of oesophagopharyngeal reflux. Such an approach would allow recognition of both defective tone and abnormal reflex relaxation of an otherwise normally contracted sphincter. Our preliminary data suggest that the latter mechanism is more likely to be the cause of oesophagopharyngeal reflux. ${ }^{14}$

The concurrent monitoring of pharyngeal and upper oesophageal body pressures provided continuous feedback on the adequacy of the sleeve position with the upper oesophageal sphincter. The sleeve length used proved more than adequate to cope with the range of sphincter movement on the recording assembly, even when the child was restless (Figs 2 and 4). Recording upper oesophageal sphincter pressure with the sleeve was made more demanding in this study by the need to combine this with simultaneous monitoring of oesophageal body, lower oesophageal sphincter, and gastric pressures. We thought that this was the only ethically acceptable approach to gathering experience with upper oesophageal sphincter manometry in children. The proportion of recording time during which technically successful dual sphincter measurements were made indicates that it is a highly efficient method for study of the integration of oesophageal motor function.

There are no data on the radial profile of upper oesophageal sphincter pressure in children. It is reasonable to assume, however, that this is asymmetric, since it has been a universal finding in animals ${ }^{2}$ and adults. ${ }^{15}$ This asymmetry is considered to be due to the muscular anatomy of the upper oesophageal sphincter, which is little different in children compared with adults. If measures of basal upper oesophageal sphincter pressure are considered to be of physiological relevance it is important to control for the influence of radial asymmetry on the pressures recorded. The oval cross sectional shape of the sleeve used in the present studies resembles that of sleeves used in adults which have been shown to orient themselves consistently in the anterior or posterior position. ${ }^{4} \mathrm{We}$ consider it reasonable to assume that the sleeve was orientated if this manner in the children that we studied and that there was consequently adequate control for radial asymmetry of the upper oesophageal sphincter pressure profile.

The experience reported in the present study indicates that it is possible to build manometric assemblies that give a great deal of information simultaneously within overall dimensions that are well tolerated by unsedated children. We believe that the sleeve method holds considerable potential for definition of patterns of upper oesophageal sphincter function in children. Smaller diameter dual sleeve assemblies are being developed which will allow application of this methodology to children in the first three months of life. Since the children in the present study had suspected oesophageal or pharyngeal dysfunction these findings cannot be considered as necessarily representative of normal. It is not yet possible to predict how valuable upper oesophageal sphincter sleeve manometry will be as an aid to the diagnosis and management of swallowing disorders and oesophagopharyngeal reflux in children. Accurate normal data need to be gathered with ethically acceptable strategies and systematic sleeve measurements of upper oesophageal sphincter function need to be made in children with well defined disorders in order to evaluate the diagnostic value of sleeve manometry in this setting.

This work was supported by a project grant from the National Health and Medical Research Council of Australia. We thank Ms J Hanley for her excellent secretarial support. We thank Drs W D A Ford, T Pouras, and D J Moore for referring their patients to us.

1 Winans $\mathrm{C}$. The pharyngoesophageal closure mechanism: a manometric study. Gastroenterology 1972; 63: 768-77.

2 Gerhardt D, Hewett J, Moeschberger M, Shuck T, Winship D. Human upper esophageal sphincter pressure profile. Am F Physiol 1980; 239: G49-52.

3 Welch RW, Drake ST. Normal lower esophageal sphincter pressure: a comparison of rapid vs. slow pull-through pressure: a comparison of rapid vs. Slow pull-

4 Kahrilas PJ, Dent J, Dodds WJ, Hogan WJ, Arndorfer RC. A method for continuous monitoring of upper esophageal sphincter pressure. Dig Dis Sci 1987; 32: 121-8.

5 Kahrilas PJ, Dodds WJ, Dent J, Logemann JA, Shaker R. Upper esophageal sphincter function during deglutition. Gastroenterology 1988; 95: 52-62.

6 Sondheimer JM. Upper esophageal sphincter and pharyngoesophageal motor function in infants with and without gastroesophageal reflux Gastroenterology 1983; 85: 301-5.

7 Staiano A, Cucciara S, De Visia B, Andreotti MR, Auriccio S. Disorders of upper esophageal sphincter motility in children. I Pediatr Gastroenterol Nutr 1987; 6: 892-8.

8 Kahrilas PJ, Dodds WJ, Dent J, Haeberle B, Hogan WJ, Arndorfer RC. Effect of sleep, spontaneous gastroesophageal reflux and a meal on upper esophageal sphincter esophageal reflux and a meal on upper esophageal sphincter
pressure in normal human volunteers. Gastroenterology 1987;

9 Asoh R, Goyal RK. Manometry and electromyography of the upper esophageal sphincter in the opossum. Gastroenterology 1978; 74: 514-20.

10 Cook IJ, Dent J, Shannon S, Collins SM. Measurement of upper esophageal sphincter pressure: effect of acute emotional stress. Gastroenterology 1987; 93: 526-32.

11 Kahrilas PJ, Dodds WJ, Dent J, Wyman JB, Hogan WJ, Arndorfer RC. Upper esophageal sphincter function during Arndorfer RC. Upper esophageal sphincter

12 Strobel CT, Byrne WJ, Ament ME, Euler AR. Correlation of esophageal lengths in children with height: application to the Tuttle test without prior esophageal manometry. $\mathcal{F}$ Pediatr Tuttle test without prior esophageal manometry. $\mathcal{F}$ Pediatr
$1979 ; 94: 81-4$. 1979; 94: 81-4.
Anvari M, Dent J, Waterfall WE, Cook I, Anvari P. Motor responses of the upper esophageal sphincter to physical straining [abstract]. Gastroenterology 1987; 92: 1297.

14 Davidson GP, Dent J, Willing J, Kocyan P. Transient upper esophageal sphincter relaxations during acid reflux in children. Gastroenterology 1988; 94: 5: 88.

15 Welch RW, Luchmann K, Ricks PM, Drake ST, Gates GA. Manometry of the normal upper esophageal sphincter and its alterations in laryngectomy. $\mathcal{F}$ Clin Invest 1979; 63: 103641. 